\title{
Insights from nanomedicine into chloroquine efficacy against COVID-19
}

\author{
Chloroquine - an approved malaria drug - is known in nanomedicine research for the investigation of \\ nanoparticle uptake in cells, and may have potential for the treatment of COVID-19.
}

\author{
Tony Y. Hu, Matthew Frieman and Joy Wolfram
}

$\mathrm{R}$ ecent multicentre clinical trials ${ }^{1}$ and cell culture studies ${ }^{2}$ suggest that the 70-year-old malaria drug, chloroquine, may potentially display therapeutic efficacy against COVID-19 (corona virus disease 2019), a rapidly spreading viral infection that can cause pneumonia-induced death in approximately $2.5 \%$ of infected individuals ${ }^{1,3}$. Based on the preliminary clinical trial findings, chloroquine has been included in federal guidelines for treatment of COVID-19 in the People's Republic of China. However, caution should be exercised when making premature interpretations, as clinical trials are still ongoing and interim trial data have not yet been made available. Given the current lack of an approved and effective vaccine for severe acute respiratory syndrome coronavirus 2 (SARS-CoV-2)', the virus causing COVID-19, it is important to evaluate potential prophylactic and/or therapeutic effects of drugs that are clinically approved for other indications. Chloroquine and its derivative, hydroxychloroquine, have a long history as safe and inexpensive drugs for use as prophylactic measures in malaria-endemic regions and as daily treatments for autoimmune diseases with the most common side effect being eye damage after long-term use ${ }^{4}$. Although previous studies have revealed that chloroquine has therapeutic activity against viruses ${ }^{5}$, including human coronavirus OC43 in animal models ${ }^{6}$ and SARS-CoV in cell culture studies ${ }^{7}$, anti-viral mechanisms of chloroquine remain speculative. Chloroquine has been used in the field of nanomedicine for the investigation of nanoparticle uptake in cells, and, therefore, insights from synthetic nanoparticle interactions with cells in the presence of chloroquine may reveal mechanisms that are active at early stages prior to viral replication. Specifically, nanomedicine studies may provide clues on chloroquine-induced alterations of SARSCoV-2 cellular uptake.

\section{Chloroquine mechanisms of action} The precise mechanisms through which chloroquine may act to attenuate

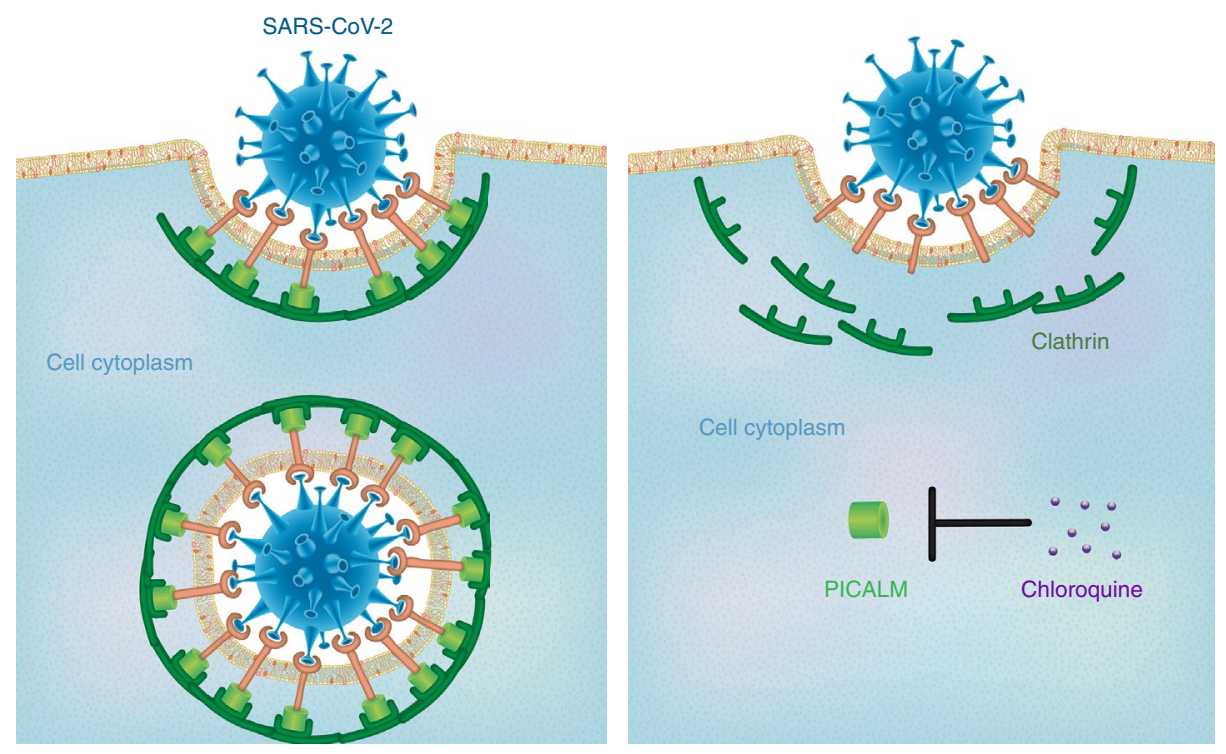

Fig. 1 | Potential mechanism by which chloroquine exerts therapeutic effects against COVID-19. The proposed mechanism involves chloroquine-induced suppression of PICALM, which prevents endocytosis-mediated uptake of SARS-CoV-2.

SARS-CoV-2 infections are of considerable interest, as this information could be valuable for identifying new prophylactic and therapeutic candidates. Chloroquine is a weak base that becomes entrapped in membrane-enclosed low $\mathrm{pH}$ organelles, interfering with their acidification ${ }^{5}$. In malaria-causing plasmodium parasites, chloroquine accumulates in the digestive vacuole where it is thought to prevent $\mathrm{pH}$-dependent detoxification of heme, which is produced upon parasitic consumption of haemoglobin to obtain free amino acids ${ }^{8}$. This sequestering effect is also apparent in mammalian cells, in which chloroquine treatment leads to an increase in lysosomal $\mathrm{pH}$. Speculation on chloroquine-induced antiviral effects include inhibition of $\mathrm{pH}$-dependent viral fusion/replication and prevention of viral envelope glycoprotein as well as host receptor protein glycosylation ${ }^{5}$. Chloroquine may also inhibit virion assembly in endoplasmic reticulum-Golgi intermediate compartment (ERGIC)-like structures. Additionally, it is probable that chloroquine exhibits host effects, independent of direct viral action, by attenuating the expression of pro-inflammatory factors and receptors that can induce acute respiratory distress syndrome, which is primarily responsible for coronavirus-associated mortality ${ }^{3}$.

\section{Chloroquine inhibits endocytosis of nanoparticles}

It has been demonstrated that chloroquine is a broad-spectrum inhibitor of nanoparticle endocytosis by resident macrophages. Therefore, chloroquine decreases the accumulation of synthetic nanoparticles of various sizes $(14-2,600 \mathrm{~nm})$ and shapes (spherical and discoidal) in cell lines, as well as in the mononuclear phagocyte system of mice in response to clinically relevant doses of chloroquine ${ }^{9,10}$. Mechanistic studies have revealed that chloroquine reduces the 
expression of phosphatidylinositol binding clathrin assembly protein (PICALM), one of the three most abundant proteins in clathrin-coated pits ${ }^{10}$. PICALM is a cargo-selecting clathrin adaptor that senses and drives membrane curvature, thereby regulating the rate of endocytosis ${ }^{11}$. Depletion of PICALM has previously been shown to inhibit clathrin-mediated endocytosis ${ }^{11}$, which is a predominant pathway for synthetic nanoparticle internalization ${ }^{10}$. Notably, protein levels of clathrin and clathrin adaptor protein 2 (AP2) were unchanged in macrophages treated with chloroquine ${ }^{10}$, indicating PICALM-specific effects, as opposed to a general reduction in proteins associated with clathrin-dependent endocytosis. Compared to chlorpromazine (a well-known clathrin-dependent endocytosis inhibitor), chloroquine was more effective in preventing nanoparticle uptake in macrophages $^{10}$, suggesting that additional mechanisms beyond suppression of clathrinmediated internalization may be involved. It is also conceivable that PICALM may play a secondary role in non-clathrinmediated nanoparticle uptake. Additionally, chloroquine is known to prevent lysosome acidification, thereby hindering fusion with endocytic vesicles ${ }^{4}$. Prevention of lysosome fusion is likely to interfere with upstream endocytic trafficking, causing a 'traffic jam' scenario that blocks effective transport of cargo to and from the cell membrane ${ }^{9}$.

\section{Potential effects of chloroquine on SARS-CoV-2}

SARS-CoV-2 falls within the same size range $(60-140 \mathrm{~nm})$ and shape (spherical) ${ }^{3}$ as commonly studied synthetic nanoparticles ${ }^{12,13}$. Therefore, it is possible that one of the mechanisms responsible for chloroquinemediated effects against SARS-CoV-2 is a general decrease in the ability of cells to perform clathrin-mediated endocytosis of nanosized structures due to PICALM suppression (Fig. 1). Other Coronaviridae are known to enter host cells through receptormediated endocytosis, although direct fusion with the plasma membrane has also been reported. For example, the SARS-CoV virus identified in 2003 and the human coronavirus NL63 (HCoV-NL63) identified in 2004 bind to the angiotensin-converting enzyme 2 (ACE2) receptor, triggering endocytosisdriven cell entry ${ }^{14,15}$. Both clathrin-mediated and clathrin/caveolae-independent endocytosis mechanisms have been described for SARS-CoV entry in human cells ${ }^{16,17}$. SARS-CoV-2 might use similar ACE2mediated mechanisms of cell entry ${ }^{18}$.

Moreover, chloroquine-induced prevention of endosome-lysosome fusion is likely to interfere with general endocytic trafficking, such as membrane receptor recycling, which is thought to be required for SARS-CoV-2 cellular entry. However, previous studies have revealed that chloroquine has therapeutic activity against SARS-CoV in cell culture but does not alter cell-surface levels of ACE2 ${ }^{7}$. Additionally, therapeutic doses of chloroquine did not substantially change the biosynthesis or glycosylation of the SARS-CoV spike glycoprotein 7 . On the contrary, terminal glycosylation of the ACE2 receptor was impaired, which may affect viral binding? Chloroquine has been shown to display antiSARS-CoV activity in cell culture even when administered after viral uptake ${ }^{7}$, suggesting that multiple beneficial mechanisms may be involved. Upon entry into cells via endocytosis, the spike protein on the surface of the virion must be cleaved by resident endosomal proteases such as cathepsins, which are activated upon acidification of the endosome. This cleavage induces a conformational change in the spike protein bringing the viral envelope and the endosomal membrane together to enable fusion. Chloroquine-induced inhibition of endosomal acidification is likely to alter this fusion event, stalling the virus in endosomes.

\section{Future studies to assess the potential of (hydroxy)chloroquine against COVID-19}

In the case that clinical trial data verify the initial findings of chloroquine activity in COVID-19 patients $^{1}$, further studies will be necessary to understand the most optimal prophylactic and/or therapeutic clinical protocols with regard to, for example, patient population, disease stage and dosing. Additionally, comparison studies should be made between chloroquine and hydroxychloroquine, as the latter is considered to have a better safety profile and was recently showed to have similar anti-SARS-CoV-2 effects in cell culture ${ }^{19,20}$. Furthermore, preclinical studies will be valuable in further determining potential (hydroxy)chloroquine-mediated anti-SARSCoV-2 mechanisms, including suppression of endocytosis in host cells. Pseudotyped virions with the SARS-CoV-2 spike would be beneficial for assessing cellular entry requirements in a simplified system, and immunofluorescence studies could identify virion location in drug-treated cells. However, caution should be taken to avoid premature interpretations of preclinical and clinical findings. In fact, chloroquine has shown therapeutic activity against Ebola virus in cell culture but animal studies have revealed conflicting results ${ }^{21,22}$. Moreover, in the case of chikungunya virus, chloroquine displayed beneficial effects in vitro, exacerbated infection in animal models, and lacked therapeutic effects, while increasing the risk of arthralgia in a clinical study ${ }^{23}$.

Other clinically approved drugs are under consideration as COVID-19 therapeutics, including human immunodeficiency virus (HIV) protease inhibitors, such as ritonavir and lopinavir. However, HIV and SARS-CoV-2 have distinct proteases, bringing into question the target specificity and usefulness of such drugs in treating COVID-19. Therapeutic agents that target host pathways or viral mechanisms that are shared among multiple viral species (for example, cellular entry or RNA genome replication) are more feasible options for causative viral agents that have not been fully characterized. Chloroquine represents a potential broad-spectrum example of inhibiting viral cell entry, while remdesivir, an investigational drug that was originally developed for Ebola virus disease, represents an example of a broad-spectrum RNA polymerase inhibitor.

There is cautious optimism that (hydroxy) chloroquine may have prophylactic and/or therapeutic effects against COVID-19, and understanding the mechanisms by which these drugs affect SARS-CoV-2 would be critical for optimizing and developing preventative and therapeutic strategies.

Tony Y. Hu ${ }^{1,7}$, Matthew Frieman ${ }^{2,7}$ and Joy Wolfram $3,4,5,6$

${ }^{1}$ Center for Cellular and Molecular Diagnostics, Department of Biochemistry and Molecular Biology, Tulane University School of Medicine, New Orleans, LA, USA. ${ }^{2}$ Department of Microbiology and Immunology, University of Maryland School of Medicine, Baltimore, MD, USA. ${ }^{3}$ Department of Biochemistry and Molecular Biology, Mayo Clinic, Jacksonville, FL, USA. ${ }^{4}$ Department of Physiology and Biomedical Engineering, Mayo Clinic, Jacksonville, FL, USA. ${ }^{5}$ Department of Transplantation, Mayo Clinic, Jacksonville, FL, USA. ${ }^{6}$ Department of Nanomedicine, Houston Methodist Research Institute, Houston, TX, USA. ${ }^{7}$ These authors contributed equally: Tony Y. Hu, Matthew Frieman. e-mail: tonyhu@tulane.edu;mfrieman@som. umaryland.edu;wolfram.joy@mayo.edu

Published online: 23 March 2020 https://doi.org/10.1038/s41565-020-0674-9

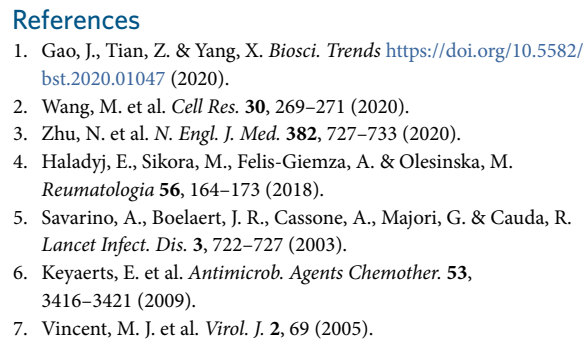

1. Gao, J., Tian, Z. \& Yang, X. Biosci. Trends https://doi.org/10.5582/

Zhu, N. et al. N. Engl. J. Med. 382, 727-733 (2020).

Lancet Infect. Dis. 3, 722-727 (2003).

7. Vincent, M. J. et al. Virol. J. 2, 69 (2005). 
8. Hempelmann, E. Parasitol. Res. 100, 671-676 (2007).

9. Pelt, J. et al. Pharmacol. Ther. 191, 43-49 (2018).

10. Wolfram, J. et al. Sci Rep 7, 13738 (2017).

11. Miller, S. E. et al. Dev. Cell 33, 163-175 (2015).

12. Wolfram, J. \& Ferrari, M. Nano Today 25, 85-89 (2019).

13. Gentile, E. et al. Future Oncol. 9, 1849-1859 (2013).

14. Wu, K., Li, W., Peng, G. \& Li, F. Proc. Natl Acad. Sci. USA 106, 19970-19974 (2009).

15. Li, W. et al. Nature 426, 450-454 (2003).

16. Wang, H. et al. Cell Res. 18, 290-301 (2008).

17. Inoue, Y. et al. J. Virol. 81, 8722-8729 (2007).

18. Wrapp, D. et al. Science 367, 1260-1263 (2020).
19. Yao, X. et al. Clin. Infect. Dis. https://doi.org/10.1093/cid/ciaa237 (2020).

20. Liu, J. et al. Cell Discov. 6, 16 (2020)

21. Dowall, S. D. et al. J. Gen. Virol. 96, 3484-3492 (2015).

22. Madrid, P. B. et al. PLoS One 8, e60579 (2013).

23. Roques, P. et al. Viruses 10, 268 (2018).

Acknowledgements

We acknowledge funding in the area of namomedicine

for infectious disease research from the following

sources: the Department of Defense under award number DODW8IXWH1910926 (T.Y.H.) and the National Institutes of Health under award numbers R01HD090927 (T.Y.H.), R01AI122932 (T.Y.H.),

R01AI113725 (T.Y.H.), R21AI126361 (T.Y.H.), R21EB026347 (T.Y.H.) and R21AI52318 (J.W.). The content is solely the responsibility of the authors and does not necessarily represent the official views of the funding agencies.

Competing interests

The authors declare no competing interests. 